\title{
Long-chain polyunsaturated fatty acids in breast milk and early weight gain in breast-fed infants
}

\author{
Salome Scholtens ${ }^{1}$, Alet H. Wijga ${ }^{2}$, Henriette A. Smit ${ }^{2,3}$, Bert Brunekreef ${ }^{1,3}$, Johan C. de Jongste ${ }^{4}$, \\ Jorrit Gerritsen $^{5}$ and Jaap C. Seidell ${ }^{6}$ \\ ${ }^{1}$ Institute for Risk Assessment Sciences (IRAS), Utrecht University, The Netherlands \\ ${ }^{2}$ Centre for Prevention and Health Services Research, National Institute for Public Health and the Environment, Bilthoven, \\ The Netherlands \\ ${ }^{3}$ Julius Centre for Health Sciences and Primary Care, University Medical Center Utrecht, The Netherlands \\ ${ }^{4}$ Department of Pediatrics, Division of Pediatric Respiratory Medicine, Erasmus University Medical Center/Sophia Children's \\ Hospital, Rotterdam, The Netherlands \\ ${ }^{5}$ Beatrix Children's Hospital, University Medical Centre Groningen, University of Groningen, The Netherlands \\ ${ }^{6}$ Department of Nutrition and Health, Faculty of Earth and Life Sciences, Vrije Universiteit, Amsterdam, The Netherlands \\ (Received 2 August 2007 - Revised 3 April 2008 - Accepted 10 April 2008 - First published online 20 May 2008)
}

The long-chain PUFA (LCPUFA) content of an infant's diet might affect early weight gain. In early trials on supplementation of formula feeding $n$-3 LCPUFA affected weight gain adversely. $n$-6 LCPUFA are thought to promote adipose tissue development and might be associated with higher weight gain. We studied the association between the natural $n-3$ and $n-6$ LCPUFA content of breast milk of Dutch women and weight and BMI gain of their breast-fed infants in the first year of life. The children in this study were enrolled in the Prevention and Incidence of Asthma and Mite Allergy (PIAMA) birth cohort study and were born in 1996-1997 in the Netherlands. Parents reported their child's weight and length in a questionnaire. Of a subgroup of the total population breast-milk samples were collected ( $n$ 244). The fatty acid composition of breast milk was determined by GLC and expressed as weight percentages. Linear regression was used for data analysis. Mean gain in weight, length and BMI per week from birth to 1 year of age was 119.5 (SD 16.1) g, 0.48 (SD 0.05) $\mathrm{cm}$ and 0.06 (SD 0.03 ) kg/m $\mathrm{m}^{2}$, respectively. The associations between $n-6$ and $n-3$ LCPUFA in breast milk, and infant weight, length and BMI gain were weak and inconsistent. The $n-3$ and $n-6$ LCPUFA content in breast milk did not affect weight or BMI gain in the first year of life in breast-fed term infants.

Breast milk: Infant growth: Long-chain PUFA: Weight gain: BMI

The long-chain PUFA (LCPUFA, derivatives of linoleic acid $(18: 2 n-6 ;$ LA) and $\alpha$-linolenic acid $(18: 3 n-3$; ALA)) content of an infant's diet might affect early growth. In particular the ratio between $n-6$ and $n-3$ LCPUFA could play a role. Early trials on supplementation of formula feeding showed that formula supplemented with $n$-3 LCPUFA affected infant growth adversely ${ }^{(1,2)}$. However, Makrides et al. ${ }^{(3)}$ concluded in a meta-analysis that supplementation of infant formula with $n$-3 LCPUFA (with or without $n$-6 LCPUFA) did not influence growth of term-infants, which was in accordance with two reviews ${ }^{(4,5)}$. Evidence from experimental and animal studies suggests that arachidonic acid $(20: 4 n-6$; AA), the main LCPUFA of the $n-6$ family, is a potent adipogenic factor that promotes adipose tissue development and possibly obesity, while $n-3$ LCPUFA could reduce an excessive increase in adipose tissue ${ }^{(6-8)}$. Furthermore, in obese subjects, a higher $n-6$ LCPUFA serum level ${ }^{(9,10)}$ and a lower $n-3$
LCPUFA level ${ }^{(11)}$ compared with normal-weight subjects has been reported.

During early development, the newborn infant is largely dependent on dietary intake for his or her $n-6$ and $n-3$ LCPUFA supply. The infant is capable of conversion of LA and ALA to their long-chain derivatives, but the enzymatic systems involved seem to be unable to supply sufficient LCPUFA to meet the requirements ${ }^{(12,13)}$. Breast-fed infants rely on the LCPUFA present in the breast milk. In general, the fatty acid composition of breast milk reflects the long-term dietary intake of fatty acids by the mother ${ }^{(14-16)}$. However, the current diet may also influence the fatty acid composition of the breast milk ${ }^{(17)}$. A close relationship has been observed between the breast-milk fatty acid composition and infant plasma and tissue fatty acids levels ${ }^{(18-20)}$.

We hypothesized that high $n-6$ LCPUFA content in breast milk is associated with higher infant weight and

Abbreviations: AA, arachidonic acid; ALA, $\alpha$-linolenic acid; LA, linoleic acid; LCPUFA, long-chain PUFA; PIAMA study, Prevention and Incidence of Asthma and Mite Allergy study.

* Corresponding author: Dr Salome Scholtens, fax + 3130274 4407, email salome.scholtens@rivm.nl 
BMI gain from birth to 1 year of age, while high $n-3$ LCPUFA content is associated with lower infant weight and BMI gain.

\section{Methods}

\section{Study design and study population}

The study population consisted of 244 mothers and children enrolled in the Prevention and Incidence of Asthma and Mite Allergy (PIAMA) birth cohort study. The children were born in 1996-1997 in the Netherlands. A detailed description of the study design has previously been published $^{(21)}$. At baseline, the study population consisted of children of 4146 mothers who were recruited from the general population during pregnancy. Data were mainly collected by means of questionnaires that contained questions on weight and length of the child, breast-feeding duration, and other maternal and child characteristics. In addition, a subsample the study population ( $n$ 1990) was selected for house visits. When the child was about 3 months of age, 1860 families agreed to participate and were visited at home. At that time, 661 of the mothers visited at home were still breast-feeding, and 276 of them were willing and able to produce a milk sample. Two children were lost to follow-up at 1 year of age. Children who were born prematurely $\left(\begin{array}{ll}n & 2\end{array}\right)$ and children of mothers who donated a milk sample when the child was younger than 6 weeks or older than 26 weeks of age $(n 7)$ were excluded from analysis. One observation was excluded due to extreme values for $n$ - 3 LCPUFA (more than nine times the standard deviation). Data on birth weight, weight at 1 year of age and length at 1 year of age were not available for twenty children, leaving 244 children available for analysis. Birth length was available for 177 children, as length at birth is often not measured in the Netherlands. Subsequently, weight gain per week from birth to 1 year of age could be calculated for 244 children, and length gain per week and BMI gain per week could be calculated for 177 children. The study protocol was approved by the medical ethics committees of the participating institutes. All parents gave written informed consent.

\section{Assessment of fatty acid composition of breast milk}

Breast-milk samples were collected either by manual expression or by breast pump, depending on the preference of the mother. The samples were put into tubes containing $2 \mathrm{ml}$ butylated hydroxyl toluene solution $(500 \mathrm{mg} / \mathrm{ml})$ to prevent oxidation. They were sent by mail to the National Institute of Public Health and the Environment and stored there at $-70^{\circ} \mathrm{C}$. Fatty acid composition of the breast milk was determined by GLC, as described by Foreman- van Drongelen et al. ${ }^{(22)}$. Details of breast-milk collection and analysis have been reported elsewhere ${ }^{(23,24)}$. In total, thirty-four individual fatty acids were measured as weight percentage of the total fat content (wt $\%$ ). Total $n$-6 LCPUFA, total $n$-3 LCPUFA, n-3:n-6 LCPUFA ratio, AA, EPA and DHA were the exposure variables of main interest. In addition, total $n-6$ PUFA, total $n$-3 PUFA, $n-3: n-6$ PUFA ratio, LA, and ALA were included as exposure variables.

\section{Outcome and confounder assessment}

In the questionnaire sent to the parents when the child was about 1 year of age the parents reported weight and length of the child the last time the child was measured. Gender, gestational age, breast-feeding duration (in weeks), maternal smoking during pregnancy (any smoking by the mother during pregnancy from 4 weeks of pregnancy), birth weight, and birth length were also reported by the parents. BMI was calculated as weight $(\mathrm{kg})$ divided by length $(\mathrm{m})$ squared. Since age of the child at reported weight and length ranged from 9 to 15 months, weight and length of the individual infants were standardized to exactly 12 months, using the average increase in weight and length per week. Weight gain, length gain and BMI gain were defined as the difference in weight, length, and BMI between birth and 1 year of age. Weight gain, length gain, and BMI gain were expressed as $\mathrm{g}$, $\mathrm{cm}$ and $\mathrm{kg} / \mathrm{m}^{2}$ per week, respectively. A high BMI gain per week in the first year of life was defined as a BMI gain above one standard deviation of the mean. Maternal pre-pregnancy BMI $\left(\mathrm{kg} / \mathrm{m}^{2}\right)$ was calculated from self-reported body weight $(\mathrm{kg})$ and height $(\mathrm{m})$.

Maternal habitual fish-intake during lactation was reported in the questionnaire administered to the parents when the child was 3 month of age. At the breast-milk collection the mothers filled in a short questionnaire and reported how often they had consumed fish in the previous week.

\section{Statistical analyses}

The individual fatty acids and groups of fatty acids were divided in tertiles and the intermediate tertile was used as reference, because we expected that the associations between the fatty acids and infant weight, length, and BMI gain were not linear. We analyzed the data using linear and logistic regression. Different fatty acids were not included simultaneously in the regression models due to high correlation between fatty acids. The association between maternal prepregnancy BMI and the fatty acid composition of breast milk was analyzed using polytomous logistic regression, with maternal pre-pregnancy BMI as exposure variable.

The following variables were considered as potential confounders: gender, age of the child at weight measurement, age of the child at breast-milk donation, method of breast-milk collection (before or between feeds), time of the day of breast-milk collection, maternal pre-pregnancy BMI, maternal smoking during pregnancy, and total breast-feeding duration. A variable was considered a confounder if inclusion of this variable in the regression model changed the association between total $n$-6 PUFA, total $n$ - 3 PUFA, total $n$ - 6 LCPUFA, or total $n-3$ LCPUFA and BMI gain by $10 \%$ or more. Age of the child at breast-milk donation and total breast-feeding duration were confounding variables, and thus included in the adjusted regression models.

The regression analyses were repeated for infants who were breast-fed for over 6 months and with inclusion of the child who was excluded due to an extreme value for $n$ - 3 LCPUFA. Furthermore, the observations included in the analyses were compared with the excluded observations. Data analysis was conducted using SAS software version 9.1 (SAS Institute, 
Inc., Cary, NC, USA). $P$ values $<0.05$ were considered to be statistically significant.

\section{Results}

The study population comprised $48 \%$ girls ( $n$ 117). In Table 1 birth weight, weight, length and BMI at 1 year and gain in weight, length and BMI in the first year of life are presented. Twenty-six children $(10.7 \%)$ had a birth weight below $3000 \mathrm{~g}$ of whom three children had a birth weight below $2500 \mathrm{~g}$. Age of the child at breast-milk donation was 15.1 (SD 3.4) weeks and ranged from 9 to 26 weeks (Table 1). In Table 2 the proportions $(\mathrm{wt} \%)$ of the various fatty acids in breast milk are shown.

The mothers who donated a breast-milk sample and who were included in the present study were, on average, more often highly educated, smoked less often during pregnancy and breast-fed longer than the breast-feeding mothers who did not. No differences were observed in gender, birth weight, and birth length of the infant.

Table 3 shows the adjusted associations between the fatty acids in breast milk and weight, length, and BMI gain per week from birth to 1 year of age. The majority of the associations were weak, were not statistically significant, and did not follow a dose-response relationship. Both low and high total n-6 LCPUFA content in breast milk were inversely associated with weight gain and BMI gain from birth to 1 year of age compared with intermediate total $n-6$ LCPUFA content, but the associations were not statistically significant. Low and high AA content was associated with lower weight gain and was significantly associated with lower BMI gain compared to intermediate AA content. Children fed breast-milk high in AA had, on average, a $4.6 \mathrm{~g}$ lower weight gain per week in the first year of life. Total $n$-6 PUFA showed a significant association with weight gain and BMI gain. Low and high total $n$-6 PUFA content was associated with lower weight gain and with lower BMI gain compared with intermediate n-6 PUFA content. No significant associations were observed between total $n$-3 LCPUFA, EPA or DHA, and weight gain, length gain and BMI gain. When high BMI gain per week in the first year of life compared with normal BMI gain was studied as an outcome variable instead of BMI gain as a continuous variable, the results were similar to the results

Table 1. Study characteristics ( $n$ 244)

(Mean values and standard deviations)

\begin{tabular}{lccc}
\hline Variable & $n$ & Mean & SD \\
\hline Birth weight $(\mathrm{g})$ & 244 & 3555 & 491 \\
Birth length $(\mathrm{cm})$ & 177 & 51.3 & 2.3 \\
BMl at birth $\left(\mathrm{kg} / \mathrm{m}^{2}\right)$ & 177 & 13.6 & 1.4 \\
Weight at 1 year $(\mathrm{g})$ & 244 & 9788 & 993 \\
Length at 1 year $(\mathrm{cm})$ & 244 & 76.2 & 2.8 \\
BMl at 1 year $\left(\mathrm{kg} / \mathrm{m}^{2}\right)$ & 244 & 16.9 & 1.3 \\
Weight gain $(\mathrm{g} /$ week) & 244 & 119.5 & 16.1 \\
Length gain $(\mathrm{cm} /$ week) & 177 & 0.48 & 0.05 \\
BMl gain $\left(\mathrm{kg} / \mathrm{m}^{2}\right.$ per week) & 177 & 0.06 & 0.03 \\
Age of infant at breast-milk & 244 & $15 \cdot 1$ & 3.4 \\
$\quad$ donation (weeks) & & & \\
Total breast-feeding duration (weeks) & 244 & 29.9 & 12.8 \\
\hline
\end{tabular}

described earlier. Adjustment for age of the infant at breastmilk collection and total breast-feeding duration did not affect the results to a large extent and did not lead to a different conclusion. The results of the analyses did not differ when the analyses were restricted to infants who were breast-fed for at least 6 months ( $n$ 140).

Maternal pre-pregnancy BMI was not significantly associated with any of the fatty acids in breast milk (data not shown).

The maternal fish-intake in the week previous to the breast milk collection corresponded to the habitual fish-intake during lactation. Fish consumption during lactation was significantly associated with the $n-3$ LCPUFA, EPA and DHA content of the breast milk but not with weight, length and BMI gain in the children (data not shown).

\section{Discussion}

We did not find evidence supporting the hypothesis that $n-6$ LCPUFA increased infant weight and BMI gain. Both high and low levels of AA, and of the precursor of AA, LA, were associated with a lower infant weight gain and BMI gain compared with an intermediate level. n-3 LCPUFA did not affect infant weight, length and BMI gain adversely in our analyses.

To our knowledge, this study is one of the first observational studies that investigates the association between the natural fatty acid composition of breast milk and infant weight, length and BMI gain. The fatty acid composition of the breast milk and the levels of $n-6$ and $n$-3 LCPUFA corresponded with data from other Dutch studies and is typical for women consuming a western diet ${ }^{(12,14,25,26)}$.

The number of subjects included in the study was thought to be adequate to detect an association, because the number of children that participated in this study was larger than in most intervention studies on the intake of LCPUFA and infant growth. Also, in an earlier study by Wijga et al. a significant association was observed between breast-milk $n-3$ LCPUFA and eczema and asthma symptoms in children using the same data ${ }^{(27)}$.

A limitation of the present study was that only one breastmilk sample was collected. Although the breast-milk fatty acid composition reflects the long-term intake by the mother ${ }^{(14-16)}$, the current diet may influence the fatty acid composition of the

Table 2. Fatty acid content (wt\%) of breast milk in Dutch mothers ( $n$ 244)

(Mean values and standard deviations)

\begin{tabular}{lrc}
\hline Fatty acids (wt\%) & Mean & SD \\
\hline Total $n$-6 PUFA & 16.197 & 4.197 \\
Total $n$-3 PUFA & 1.530 & 0.429 \\
Total $n$-6 LCPUFA & 1.126 & 0.276 \\
Total $n$-3 LCPUFA & 0.514 & 0.210 \\
$n-3: n-6$ PUFA & 0.099 & 0.031 \\
$n-3: n-6$ LCPUFA & 0.471 & 0.197 \\
Linoleic acid $(18: 2 n-6)$ & 15.070 & 4.089 \\
$\alpha-$ Linolenic acid $(18: 3 n-3)$ & 1.016 & 0.375 \\
Arachidonic acid $(20: 4 n-6)$ & 0.370 & 0.092 \\
EPA $(20: 5 n-3)$ & 0.050 & 0.042 \\
DHA $(22: 6 n-3)$ & 0.195 & 0.128
\end{tabular}

LCPUFA, long-chain PUFA. 
Table 3. Adjusted associations* between tertiles of fatty acids (intermediate tertile is reference) in breast milk and weight gain ( $n$ 244), length gain and BMI gain $(n 177)$ in breast-fed infants per week from birth to 1 year of age

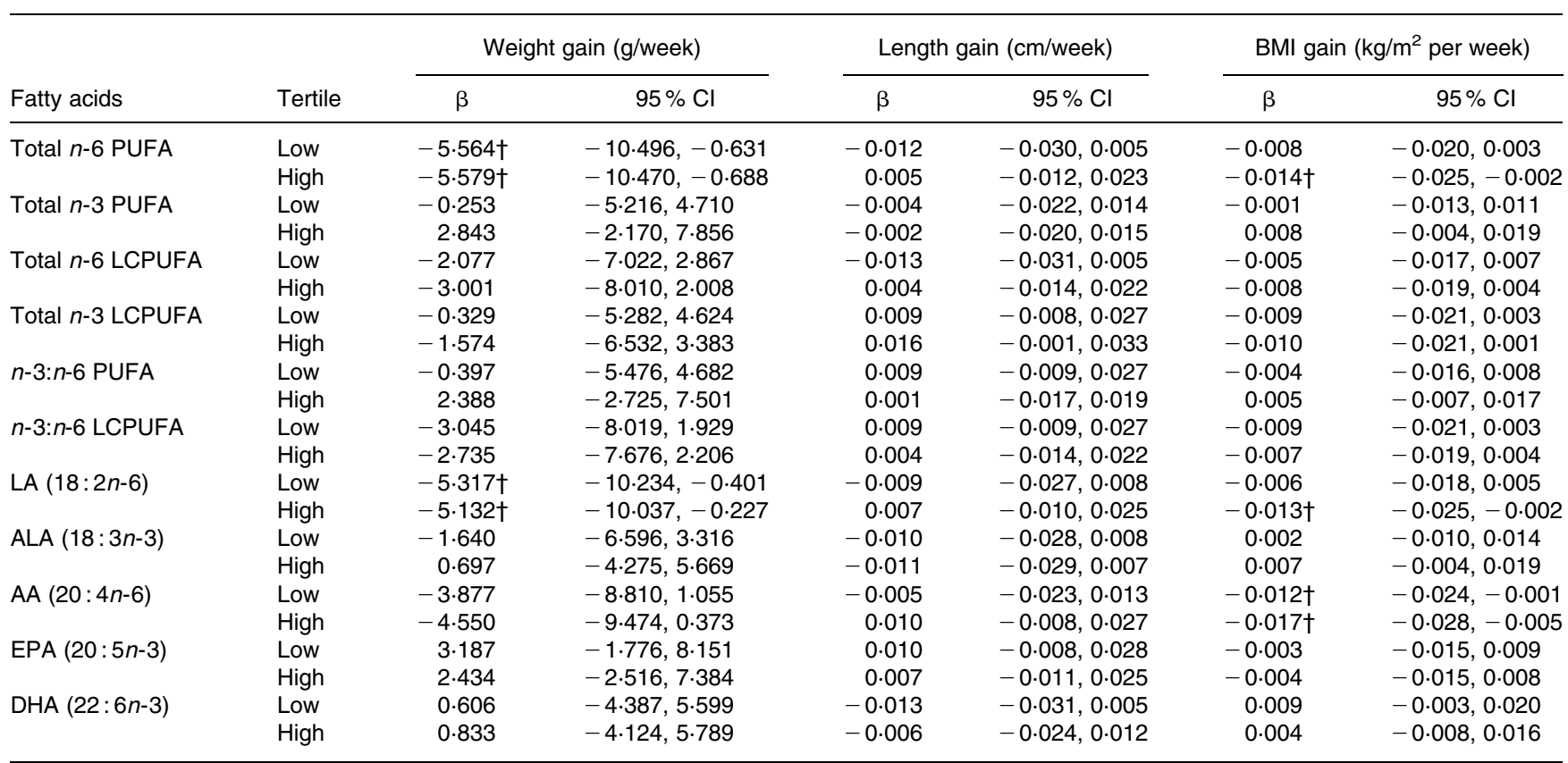

LA, linoleic acid; ALA, $\alpha$-linolenic acid; AA, arachidonic acid.

${ }^{*}$ Regression models contain the exposure variables (low and high fatty acid tertiles), age of child at breast-milk collection and total breast-feeding duration. $\dagger P<0.05$.

breast-milk also ${ }^{(17)}$. Since we collected only one breast-milk sample we cannot be certain that the fatty acid composition in the breast-milk sample reflects the fatty acid composition of the breast milk of the entire lactation period. However, we did not expect that the maternal diet during lactation changed to an extent that it would influence the results of this study. For instance, the maternal fish-consumption in the week previous to the breast-milk collection corresponded with habitual fish intake.

It is not likely that the infant's fatty acid intake via other foods than breast milk influenced the association between fatty acid intake by breast milk and weight gain, since the infants were breast-fed for 30 weeks on average and other foods make up only a small part of the diet in infancy. Besides, even if the infants received some LCPUFA via foods, all children did and probably in similar quantities. Therefore, it is unlikely that LCPUFA from another source than breast milk would have distorted the association between the fatty acid composition of the breast milk and infant weight gain.

Another limitation was that the breast-milk samples were not collected at the same age of the infant. However, the fatty acids composition differed only slightly with age of the child at breast-milk collection and we included age of the child at collection as a confounder in our analyses. Furthermore, the mothers were free to choose when to collect the sample (before, during or after the feeding) and at which time of the day. Analyses of the method and time of collection of the breast milk showed no differences in fatty acids content. Another limitation was that age of the child at weight and length measurement differed between children. We tried to overcome this problem by standardizing weight, length and
BMI to 12 months of age. Although, weight and length of the child were reported by the parents, it is not likely that this caused bias. In the Netherlands, infants are weighed and measured regularly in the first year of life in under-five-clinics and parents receive a booklet recording weight and length of their child. It is unlikely that parents underreported or overreported weight or length of their child at such a young age. Parental reported pre-pregnancy weight of the mother, on the other hand, could be underestimated. Since this underestimation is probably not associated with the fatty acid composition of the breast milk, it is unlikely that it has influenced the direction of our results, though it might have weakened our associations. Since only mothers who were still breast-feeding during the house visit were eligible for this study, some selection bias might have occurred. For instance, the mothers in this sample were higher educated than the total study population. However, we do not have reason to expect that the observed associations are different in the total population.

The results of our study correspond with the conclusions of meta-analyses on the effect of supplementation of formula feeding with LCPUFA on weight and length gain in term infants ${ }^{(3-5)}$. They reported no association between $n-3$ and n-6 LCPUFA and weight and length gain in term infants. Also, a study that investigated the association between erythrocyte LCPUFA levels in French and Mauritian infants and anthropometric parameters observed no difference in weight and length at 6 weeks of age ${ }^{(19)}$. Weight and length of infants of mothers receiving a supplement rich in $n$-3 LCPUFA during lactation, which did increase the $n-3$ LCPUFA content in the mothers' breast milk, did not differ in the first year of life ${ }^{(28,29)}$. Lauritzen et al. ${ }^{(28)}$, observed a higher BMI at 2.5 years of age in the children of Danish mothers who had 
received n-3 LCPUFA supplementation during lactation. However, a German study showed a lower BMI at 21 months in children of mothers supplemented with DHA during lactation $^{(30)}$. Since the association between $n-3$ LCPUFA and BMI gain was very small in our observational study, we do not expect that a longer follow-up would reveal a positive association between $n-3$ LCPUFA and BMI. A study in Chinese infants living in a rural area reported a positive association between AA in breast milk and weight gain in the first 3 months of life and a positive association between AA and DHA in breast milk and length gain in the first 3 months of life ${ }^{(31)}$. This study included a small study sample (twenty-three infants at 3 months) and the breast milk $n-6$ PUFA content was considerably higher than in our study.

An inverse association was observed between a high breast milk $n-6$ LCPUFA content, and infant weight and BMI gain. $n$-6 LCPUFA derive from $n$-6 PUFA, in particular LA. $n-6$ PUFA were also associated with a lower weight and BMI gain. The reason for the lower weight gain in infants fed breast-milk containing a high $n$-6 LCPUFA or $n$ - 6 PUFA content remains unclear. The fatty acid composition of breast milk reflects the long-term dietary intake of fatty acids by the mother ${ }^{(14-16)}$. Mothers whose breast milk is high in $n-6$ LCPUFA and $n-6$ PUFA, most likely have a diet that is high in $n-6$ PUFA. Possibly other nutrients associated with the consumption of a diet high in $n-6$ PUFA might influence infant weight and BMI gain.

In conclusion, we did not observe a consistent association between $n-3$ and $n-6$ LCPUFA in breast milk and weight and BMI gain in the first year of life in breast-fed term infants.

\section{Acknowledgements}

The study was supported by the Netherlands Organisation for Health Research and Development, the Netherlands Asthma Foundation, the Netherlands Ministry of Health, Welfare and Sport and Numico Research, the Netherlands. The funding source had no involvement in the study-design, collection of the data, data-analysis, interpretation of the data, writing the report and nor in the decision to submit the paper for publication.

The authors declare that there is no conflict of interest.

\section{References}

1. Carlson SE, Werkman SH \& Tolley EA (1996) Effect of longchain $n$-3 fatty acid supplementation on visual acuity and growth of preterm infants with and without bronchopulmonary dysplasia. Am J Clin Nutr 63, 687-697.

2. Ryan AS, Montalto MB, Groh-Wargo S, et al. (1999) Effect of DHA-containing formula on growth of preterm infants to 59 weeks postmenstrual age. Am J Human Biol 11, 457-467.

3. Makrides M, Gibson RA, Udell T \& Ried K (2005) Supplementation of infant formula with long-chain polyunsaturated fatty acids does not influence the growth of term infants. Am J Clin Nutr 81, 1094-1101.

4. Lapillonne A \& Carlson SE (2001) Polyunsaturated fatty acids and infant growth. Lipids 36, 901-911.

5. Gibson RA, Chen W \& Makrides M (2001) Randomized trials with polyunsaturated fatty acid interventions in preterm and term infants: functional and clinical outcomes. Lipids 36, $873-883$.
6. Ailhaud G, Massiera F, Weill P, Legrand P, Alessandri JM \& Guesnet P (2006) Temporal changes in dietary fats: role of $n-6$ polyunsaturated fatty acids in excessive adipose tissue development and relationship to obesity. Prog Lipid Res 45, 203-236.

7. Gaillard D, Negrel R, Lagarde M \& Ailhaud G (1989) Requirement and role of arachidonic acid in the differentiation of preadipose cells. Biochem J 257, 389-397.

8. Massiera F, Saint-Marc P, Seydoux J, et al. (2003) Arachidonic acid and prostacyclin signaling promote adipose tissue development: a human health concern? J Lipid Res 44, 271-279.

9. Decsi T, Molnar D \& Koletzko B (1996) Long-chain polyunsaturated fatty acids in plasma lipids of obese children. Lipids $\mathbf{3 1}$, 305-311.

10. Savva SC, Chadjigeorgiou C, Hatzis C, et al. (2004) Association of adipose tissue arachidonic acid content with BMI and overweight status in children from Cyprus and Crete. $\mathrm{Br} J$ Nutr 91, 643-649.

11. Klein-Platat C, Drai J, Oujaa M, Schlienger JL \& Simon C (2005) Plasma fatty acid composition is associated with the metabolic syndrome and low-grade inflammation in overweight adolescents. Am J Clin Nutr 82, 1178-1184.

12. Lauritzen L, Hansen HS, Jorgensen MH \& Michaelsen KF (2001) The essentiality of long chain $n-3$ fatty acids in relation to development and function of the brain and retina. Prog Lipid Res 40, 1-94.

13. Szitanyi P, Koletzko B, Mydlilova A \& Demmelmair H (1999) Metabolism of 13C-labeled linoleic acid in newborn infants during the first week of life. Pediatr Res 45, 669-673.

14. Jensen RG (1999) Lipids in human milk. Lipids 34, 1243-1271.

15. Demmelmair H, Baumheuer M, Koletzko B, Dokoupil K \& Kratl G (1998) Metabolism of U13C-labeled linoleic acid in lactating women. J Lipid Res 39, 1389-1396.

16. Del Prado M, Villalpando S, Elizondo A, Rodriguez M, Demmelmair H \& Koletzko B (2001) Contribution of dietary and newly formed arachidonic acid to human milk lipids in women eating a low-fat diet. Am J Clin Nutr 74, 242-247.

17. Innis SM (2007) Human milk: maternal dietary lipids and infant development. Proc Nutr Soc 66, 397-404.

18. Mellies MJ, Ishikawa TT, Gartside PS, et al. (1979) Effects of varying maternal dietary fatty acids in lactating women and their infants. Am J Clin Nutr 32, 299-303.

19. Pugo-Gunsam P, Guesnet P, Subratty AH, Rajcoomar DA, Maurage C \& Couet C (1999) Fatty acid composition of white adipose tissue and breast milk of Mauritian and French mothers and erythrocyte phospholipids of their full-term breast-fed infants. Br J Nutr 82, 263-271.

20. Jensen CL, Maude M, Anderson RE \& Heird WC (2000) Effect of docosahexaenoic acid supplementation of lactating women on the fatty acid composition of breast milk lipids and maternal and infant plasma phospholipids. Am J Clin Nutr 71, 292S-299S.

21. Brunekreef B, Smit J, de Jongste J, et al. (2002) The prevention and incidence of asthma and mite allergy (PIAMA) birth cohort study: design and first results. Pediatr Allergy Immunol 13, Suppl. 15, 55-60.

22. Foreman-van Drongelen MM, Houwelingen AC, Kester AD, et al. (1995) Long-chain polyene status of preterm infants with regard to the fatty acid composition of their diet: comparison between absolute and relative fatty acid levels in plasma and erythrocyte phospholipids. Br J Nutr 73, 405-422.

23. Wijga A, Houwelingen AC, Smit HA, et al. (2003) Fatty acids in breast milk of allergic and non-allergic mothers: The PIAMA birth cohort study. Pediatr Allergy Immunol 14, 156-162.

24. van der Steege G, Muskiet FA, Martini IA, Hutter NH \& Boersma ER (1987) Simultaneous quantification of total mediumand long-chain fatty acids in human milk by capillary gas chromatography with split injection. J Chromatogr 415, 1-11. 
25. Dagnelie PC, van Staveren WA, Roos AH, Tuinstra LG \& Burema J (1992) Nutrients and contaminants in human milk from mothers on macrobiotic and omnivorous diets. Eur $J$ Clin Nutr 46, 355-366.

26. Huisman $M$, van Beusekom CM, Lanting CI, Nijeboer HJ, Muskiet FA \& Boersma ER (1996) Triglycerides, fatty acids, sterols, mono- and disaccharides and sugar alcohols in human milk and current types of infant formula milk. Eur J Clin Nutr 50, 255-260.

27. Wijga Alet $\mathrm{H}$, van Houwelingen Adriana C, Kerkhof Marjan, Tabak Cora, de Jongste Johan C \& Smit Henriette A (2006) Breast milk fatty acids and allergic disease in preschool children: The Prevention and Incidence of Asthma and Mite Allergy birth cohort study. J Allergy Clin Immunol 117, 2, 440-447.
28. Lauritzen L, Hoppe C, Straarup EM \& Michaelsen KF (2005) Maternal fish oil supplementation in lactation and growth during the first 2.5 years of life. Pediatr Res 58, 235-242.

29. Helland IB, Saugstad OD, Smith L, et al. (2001) Similar effects on infants of $n-3$ and $n-6$ fatty acids supplementation to pregnant and lactating women. Pediatrics 108, E82.

30. Lucia Bergmann R, Bergmann KE, Haschke-Becher E, et al. (2007) Does maternal docosahexaenoic acid supplementation during pregnancy and lactation lower BMI in late infancy? $J$ Perinat Med 35, 295-300.

31. Xiang M, Lei S, Li T \& Zetterstrom R (1999) Composition of long chain polyunsaturated fatty acids in human milk and growth of young infants in rural areas of northern China. Acta Paediatr 88, 126-131. 